\title{
ESTIMATION OF COLLECTIVE INSTABILITIES IN RHIC *
}

\author{
W.W. MacKay, M. Blaskiewicz, D. Deng, V. Mane, S. Peggs, \\ A. Ratti, J. Rose, T. J. Shea, J. Wei, \\ Brookhaven National Laboratory, Upton NY, 11973-5000
}

\begin{abstract}
We have estimated the broadband impedance in RHIC to be $|Z / n|<1.2 \Omega$ for frequencies above $100 \mathrm{MHz}$. The $Z / n$ threshold is set for $\mathrm{Au}^{+79}$ ions at transition with an estimated $10 \%$ growth in emittance for $Z / n=1.5 \Omega$. We summarize the sources of broad and narrow band impedances in RHIC and investigate the multibunch instability limits throughout the machine cycle. The largest contribution to the broadband impedance comes from the abort and injection kickers (see [1]). Since RHIC is designed to accelerate fully stripped ions from $\mathrm{H}^{+}$up to $\mathrm{Au}^{+79}$ we give results for both protons and gold ions; other ions should give results somewhere between these two extremes. All ion species are expected to be stable during storage. At lower energies damping systems and chromaticity corrections will limit any growth to acceptable levels during the short time it takes to inject and accelerate the beams.
\end{abstract}

\section{INTRODUCTION}

The Relativistic Heavy Ion Collider (RHIC) is designed (see [2]) to collide fully stripped ions from protons to gold, at a maximum energy of $250 \mathrm{GeV}$ (protons) or $100 \mathrm{GeV} / \mathrm{u}\left(\mathrm{Au}^{+79}\right)$. Counter-rotating beams of particles will collide head-on at up to six interaction regions. The two intersecting superconducting rings have a circumference of $3.834 \mathrm{~km}$ with a maximum revolution frequency, $f_{\text {rev }}=78.2 \mathrm{kHz}$. The rf system consists of a set of $26.7 \mathrm{MHz}$ cavities for injection and acceleration, and a set of $196 \mathrm{MHz}$ cavities for storage. Some typical values of parameters including tunes, chromaticities, and emittances are given in Table I.

Table I. Typical operating parameters.

$\begin{array}{lcc}\gamma(\mathrm{p}) & 31.2 & 268.2 \\ \gamma(\mathrm{Au}) & 12.6 & 108.4 \\ Q_{x} & 28.18 & 28.18 \\ Q_{y} & 29.18 & 29.18 \\ f_{s y n c}(\mathrm{p}) & 55 \mathrm{~Hz} & 333 \mathrm{~Hz} \\ f_{\text {sync }}(\mathrm{Au}) & 121 \mathrm{~Hz} & 388 \mathrm{~Hz} \\ d Q_{x, y} / d \delta(\mathrm{p}) & \sim 2 & \sim 2 \\ d Q_{x, y} / d \delta(\mathrm{Au}) & \sim-3 & \sim 2 \\ \sigma_{p} / p(\mathrm{p}) & 0.0005 & 0.0003 \\ \sigma_{p} / p(\mathrm{Au}) & 0.0003 & 0.0005 \\ \pi \epsilon_{95 \%}^{\mathrm{N}}(\mathrm{p}) & 15 \pi \mu \mathrm{m} & 15 \pi \mu \mathrm{m} \\ \pi \epsilon_{95 \%}^{\mathrm{N}}(\mathrm{Au}) & 10 \pi \mu \mathrm{m} & 15 \pi \mu \mathrm{m}\end{array}$

The machine cycle may be broken into four phases: injection, acceleration, rebucketing, and storage. Vulnerability to instabilities is greatest during injection, storage, and transition crossing.

\footnotetext{
*Work performed under the auspices of the US DoE.
}

Injection of 54 bunches $\left(\sim 10^{11}\right.$ nucleons per bunch) into both rings is expected to take about $30 \mathrm{~s}$ for protons and about $120 \mathrm{~s}$ for other ions. Ion species other than protons will cross transition during the acceleration part of the cycle, when the Lorentz factor $\gamma=\gamma_{\mathrm{t}}=22.89$. Rebucketing occurs at top energy when beam is transferred from the $26.7 \mathrm{MHz}$ rf buckets used for injection and acceleration, to the $196 \mathrm{MHz}$ buckets used for storage.

\section{BROADBAND IMPEDANCES}

The dominant contribution to broadband impedance is expected to be from the injection kicker magnets. There are four $1.1 \mathrm{~m}$ long injection kickers (see $[3,4]$ ) in each ring. Each module is constructed from alternating ferrite and ceramic $\mathrm{C}$-shaped elements with an inner and outer conductor. Inside the kicker is a ceramic beam pipe with a $41.3 \mathrm{~mm}$ diameter aperture. With no shielding added to the ceramic pipes, $|Z / n|<0.25 \Omega$. The ®ve abort kickers in each ring have larger apertures and hould make a smaller contribution to the overall impedance; however, for impedance estimation we have assumed that they give a contribution identical to the injection kickers. We are actively pursuing the use of image current strips on the ceramic beam pipes to reduce the impedances of these elements.

Over three quarters of the beam pipe circumference is cold, with an inner diameter of $6.9 \mathrm{~cm}$. For the most part the remainder is warm with an aperture of $12.3 \mathrm{~cm}$. The beampipe cutoff frequency for TM modes is $3.3 \mathrm{GHz}$. The resistive wall impedance versus frequency is estimated to be

$$
Z^{\|}(f)=(1-i) f^{1 / 2} 748 \Omega[\mathrm{GHz}]^{-1 / 2} .
$$

The beam position monitors contribute about $-0.6 i \Omega$ to $Z / n$ at very low frequencies $(\ll 0.65 \mathrm{GHz})$. For high frequencies the monitors contribute as

$$
\left|Z^{\|} / n\right| \leq 0.12 f^{-1}[\Omega \mathrm{GHz}] \text {, for } f \gg 0.65 \mathrm{GHz} .
$$

Since unshielded bellows would contribute $\sim 1 \Omega$ to $|Z / n|$, almost all of the bellows are to be shielded, resulting in an estimated contribution of only $0.02 \Omega$. Vacuum ports, gate valves, and collimators will also be shielded, and most pipe transitions will be tapered with a transition length at least $®$ ve times as long as the change in pipe radius.

\section{RF CAVITY IMPEDANCES}

The accelerating and storage rf systems (see [5]) have narrow band cavities with high shunt impedances which contribute to coupled-bunch instabilities, requiring the design of passive higher order mode dampers to detune the high $Q$ resonances. The rf system must be capable of capturing, accelerating, and storing for 10 hours the nominal load of 54 bunches of particles with an average current of $70 \mathrm{~mA}$. For each ring there are two 
26.7 MHz cavities for injection and acceleration. There are also seven $196 \mathrm{MHz}$ cavities per ring for storage. (Four are common to both rings.) An additional broadband system will be used to damp out injection momentum errors.

\section{FEEDBACK AND DAMPING SYSTEMS}

The transverse damping system will employ strip-line kickers which produce both electric and magnetic de-ection and can support a wide system bandwidth capable of damping individual bunches. The maximum expected injection errors will lead to betatron oscillations with an amplitude of $2 \mathrm{~mm}$. To avoid emittance dilution of the beam, a damping time equivalent to $100 \mathrm{rev}-$ olutions ( $1.3 \mathrm{~ms})$ is required. Transverse head-tail modes could be damped with a minimal upgrade to the feedback circuits.

Longitudinal emittance blowup can occur during injection when a bunch injected off-center in a bucket starts a dipole oscillation. The bunches in each AGS cycle are transferred into the RHIC rings one by one. The acceptable uncorrected injection $\Delta p / p$ error is $10^{-5}$ for negligible longitudinal emittance blowup. However, it is quite possible that the errors will be as large as $10^{-4}$ which, if uncorrected, would lead to a $30 \%$ blowup for a $0.2 \mathrm{eVs} / \mathrm{u}$ gold beam $(95 \%$ emittance). This higher limit sets a goal for the damping system. Simulations show that the damping time is approximately $5 \tau_{\mathrm{s}}=0.05 \mathrm{~s}$ at $1 \mathrm{kV}$ per kick, and that the resultant emittance blowup at the end of damping is only $5 \%$.

\section{STABILITY CALCULATIONS}

For purposes of stability calculations we consider protons and fully stripped gold ions. The other species are expected to lie somewhere in between these two cases.

Beam-induced @elds with wavelengths short compared to the bunch length may induce microwave instabilities in a single bunch. For a speci®ed beam intensity, this phenomenon imposes a limit on the longitudinal impedance, $\left|Z^{\|} / n\right|$, within the frequency range from the average bunch-spectrum frequency of $400 \mathrm{MHz}$ to the beampipe cut-off frequency of about $3.3 \mathrm{GHz}$.

Intrabeam scattering (IBS) will cause a bunch of $10^{11}$ protons to grow from 0.3 to $0.7 \mathrm{eV} \cdot \mathrm{s}$ during a 10 hour storage (see [8]). For gold ions after 10 hours, the intensity will have dropped $40 \%$ from IBS, and the longitudinal emittance will be about $1 \mathrm{eV} \cdot \mathrm{s}$.

The microwave threshold $|Z \| / n| \simeq 2.1 \Omega$ is set by protons at the top end of acceleration before switching to the $196 \mathrm{MHz}$ rf system. A lower limit of $1.5 \Omega$ results from limiting longitudinal emittance growth to $10 \%$ for gold ions crossing transition in the presence of a $\gamma_{\mathrm{t}}$-jump. The total broadband impedance is less than $\sim 1 \Omega$ at $400 \mathrm{MHz}$ and drops to $0.6 \Omega$ at $3 \mathrm{GHz}$.

Longitudinal coupled-bunch instability growth rates were calculated analytically following Baartman (see [9]) and compared with results obtained by the code ZAP[10]. Impedance limits for the $26.7 \mathrm{MHz}$ accelerating cavities were set by limiting the growth rate to $2 \mathrm{~s}^{-1}$, which is a factor of @ve below the capability of the damping system. Higher order mode dampers are being developed to satisfy this requirement. During storage, longitudinal coupled-bunch instabilities are expected to be Landau damped.
It is not as straightforward to apply transverse instability theory to RHIC as it is to apply longitudinal instability theory. Calculations are easiest in the weak-coupling limit, when the frequency shift of a mode is small compared to the synchrotron frequency. This is not always the case for RHIC, especially at low energies during injection. On the other hand, calculations (see [11]) which include mode coupling and which are not in the weak-coupling limit are not yet fully reliable. At low energies the space-charge tune shift (see [12]) is large compared to the synchrotron tune, further complicating mode coupling calculations.

Using the ZAP formalism in the weak-coupling limit, we obtain a maximum growth rate of $27 \mathrm{~s}^{-1}$ for protons at injection with zero chromaticity and no transverse damper. Halfway up the acceleration ramp the growth rate for protons drops to $5.6 \mathrm{~s}^{-1}$. Gold gives slightly lower values. Increasing (decreasing) the chromaticity above (below) transition can greatly decrease the growth rates, and with a slight amount of help from the transverse dampers, growth can be virtually eliminated for both protons and gold ions.

During storage, the largest contribution to impedance is due to the transverse space-charge effect: $8 \mathrm{M} / \mathrm{m}$ and $19 \mathrm{M} \Omega / \mathrm{m}$ for protons and gold, respectively. Here the resulting tune shifts are small compared to the synchrotron tune, $Q_{s} \simeq 0.005$. Any coherent motion should be Landau damped as long as the betatron tune spread is at least twice as large as the space-charge tune shifts calculated when the tune spread is neglected (see [10]). Multiplying by a safety factor of two results in a minimum rms betatron tune spread of 0.002 , which is reasonable to expect in practice.

The head-tail instability (see [13]) couples the longitudinal and transverse motion of a single bunch together. Although practically all storage rings observe this phenomenon, it is universally found to depend strongly on the horizontal and vertical chromaticities. We expect that a chromaticity of -3 units below transition and +2 units above transition should ensure stability. A slew rate of 5 units in $30 \mathrm{~ms}$ will be achievable by the RHIC sextupoles. This will allow a complete chromaticity shift at transition crossing within one synchrotron oscillation period. (The use of a transition tune jump minimizes the crossing to only 1300 turns.) In reality it would take several synchrotron oscillation periods for a head-tail instability to set in.

\section{CONCLUSIONS AND COMMENTS}

The RHIC narrowband impedance spectrum is dominated by rf cavities. The resonances from the $26.7 \mathrm{MHz}$ cavities used during injection and acceleration are somewhat stronger than those from the $196 \mathrm{MHz}$ cavities used during storage.

The broadband impedance spectrum is dominated by the resistive wall effect at very low frequencies and by kicker magnets in the intermediate frequency range up to $3 \mathrm{GHz}$.

The threshold for longitudinal microwave instabilities is lowest for protons during rebucketing, with a $|Z \| / n|$ broadband impedance limit of $2.1 \Omega$ in the frequency range $400 \mathrm{MHz}$ to $3 \mathrm{GHz}$. If we limit the longitudinal emittance growth for gold ions at transition to about $10 \%$, the broadband impedance limit then becomes $1.5 \Omega$. 
Longitudinal coupled-bunch growth rates are worst for protons at injection. Calculations show that the passive higher order mode dampers on the $26.7 \mathrm{MHz}$ cavities will limit the worst case growth rate to $2 \mathrm{~s}^{-1}$, well within the range of the active longitudinal damping system that will have a maximum damping rate of $10 \mathrm{~s}^{-1}$. At storage the $196 \mathrm{MHz}$ cavities will give a worst case growth rate of less than $1 \mathrm{~s}^{-1}$, well within the parameters of the active damping system, if not Landau damped.

Transverse coupled-bunch growth rates are also worst for protons at injection, when the resistive wall impedance may lead to a growth rate of about $30 \mathrm{~s}^{-1}$. The transverse damper system is designed to handle growth rates up to $720 \mathrm{~s}^{-1}$ and so will easily stabilize any emittance growth from the resistive wall instability at injection and during acceleration. At storage the beams will be Landau damped. Calculations performed in the weak coupling limit show that the stored beam is stabilized against transverse coupled-bunch instabilities by adjusting the chromaticity.

Space charge is a very signi ${ }^{\circledR}$ cant effect, since it places a limit on the longitudinal impedance for gold ions at transition. Space charge also produces a tune spread that can be much larger than the synchrotron tune, especially at injection energies. Calculations of mode coupling instabilities in this regime are less reliable than in the traditional weak coupling regime. We continue to develop the strong coupling model and to compare our results with other machines.

All species of ion beams are expected to be stable during storage. At lower energies the damping systems and chromaticity corrections will limit any growth to acceptable levels during the short time it takes to get from injection to storage.

\section{References}

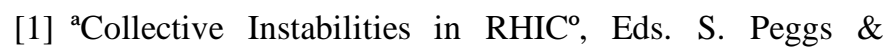
W. W. MacKay, RHIC/AP/36 (1994).

[2] ${ }^{a}$ RHIC Design Manual ${ }^{\circ}$.

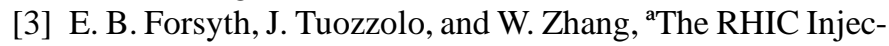
tion Fast Kicker ${ }^{\circ}$, these proceedings.

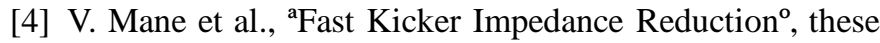
proceedings.

[5] J. Rose et al., ${ }^{\mathrm{R} F}$ Systems for $\mathrm{RHIC}^{\mathrm{o}}$, these proceedings.

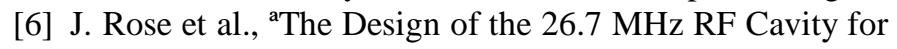
$\mathrm{RHIC}^{\mathrm{o}}$, these proceedings.

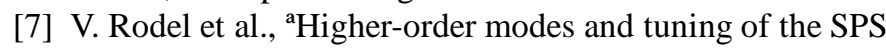
$200 \mathrm{MHz}$ single-cell cavity ${ }^{\circ}$, CERN SL/RFS/91-08, (1991).

[8] J. Wei, ${ }^{a}$ Evolution of a Hadron Beam Under IBS ${ }^{\circ}$, p. 3651, Proc. of PAC93, Washington (1993).

[9] R. Baartman, ${ }^{\mathrm{a}} \mathrm{Effect}$ of the Beam on $\mathrm{RF}^{\circ}$, US Particle Accelerator School, Florida State University, (1992).

[10] M. Zisman et al., 'aAP User's Manual ${ }^{\circ}$, LBL-21270 UC28, (1986).

[11] M. Blaskiewicz and W. T. Weng, Phys. Rev. E $\underline{50}, 4030$ (1994).

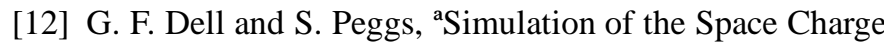
Effect in RHIC ${ }^{\circ}$, these proceedings.

[13] S. Peggs and V. Mane, ${ }^{a}$ KRAKEN, a Numerical Model of RHIC Impedances ${ }^{\circ}$, these proceedings. 